\title{
Modèle physico-chimique de cellule excitable
}

\author{
RenÉ GIBERT \\ Laboratoire de Chimie-Pbysique, Faculté des Sciences, \\ Université de Nancy, Nancy, France
}

\begin{abstract}
Physico-chemical model of the excitable cell. A simple physico-chemical model of the excitable cell is represented by a peculiar chemically active ion exchange medium. It reveals some properties of natural cells: chemical and electrical irritability, all or none effect, summation of stimuli, electrotonus, refractory periods, periodical oscillations, action potentials, conduction of impulse. This model is based upon oversimplified assumptions, and a further investigation into more details is required.
\end{abstract}

\section{INTRODUCTION}

Ce mémoire propose un modèle physico-chimique simple qui reproduit un certain nombre de phénomènes observés classiquement sur la cellule excitable. Il résulte du développement d'idées antérieures (GIBERT 1952,1953) sous une forme améliorée et plus détaillée.

Il présente par rapport aux théories connues (BERNSTEIN 1902, HILL 1935, Hodgrin 1963, Monnier 1934, Polissar 1954, Rasheysky 1933, 1950) des différences qui paraissent justifier une confrontation avec les résultats de l'experimentation plus approfondie que celle qui est amorcée ici. Ce modèle est construit sur deux hypothèses:

(1) Une image simplifiée à l'extrême de la cellule qui est assimilée à un échangeur d'ions (milieu intérieur) plongé dans une solution d'électrolyte diffusible (milieu extérieur). On peut admettre provisoirement que l'on situe ainsi le siège des phénomènes essentiels de l'excitabilité dans la membrane.

L'étude des membranes ioniques a débuté avec les mémoires de Teorell (1935) et de Meyer \& Sievers (1936). Son développement considérable a pour conséquence une littérature extrêmement touffue. Nous renverrons de lecteur a l'ouvrage d'ensemble d'HeLLFERICH (1963) pour les justifications nécessaires.

(2) Le milieu intérieur est le siège d'une réaction libératrice d'énergie. La migration des ions diffusibles à la frontière des deux milieux sous l'influence des variations de concentration ou de polarisation peut modifier cette réaction, et inversement.

Comme toutes les hypothèses simplificatrices que l'on peut admettre dans un domaine aussi complexe, ces deux conceptions sont évidemment discutables et certaine- 
ment provisoires. Mais les résultats, que, dans leur simplicité, elles permettent d'obtenir, paraissent justifier une étude plus détaillée.

Nous analyserons successivement ces deux hypothèses en commençant par la deuxième et nous en déduirons quelques conséquences.

\section{HYPOTHESE CHIMIQUE}

On admet que le milieu intérieur peut recevoir de l'énergie d'une réaction chimique exoénergétique, représentée symboliquement par l'équation:

$$
\mathrm{M} \rightarrow \mathrm{N}+\mathrm{P}+\mathrm{Q}_{\mathrm{cal}}
$$

qui traduit la décomposition du corps $M$ en ses produits de dégradation $N$ et $P$.

On laisse en dehors de l'étude les réactions qui aboutissent au corps $\mathrm{M}$, ainsi que celles que peuvent subir ultérieurement $N$ et $P$. On admet que le mécanisme de cette réaction fait intervenir deux catalyseurs: un ion $\mathrm{X}^{-}$et une molécule neutre $\mathrm{U}$. Ces catalyseurs interviennent dans la synthèse de deux ions intermédiaires $\mathrm{Z}^{-}$et $\mathrm{Y}^{\sim}$. L'ion $\mathrm{Z}^{-}$est produit par la réaction du catalyseur $\mathrm{X}^{-}$sur le corps initial $\mathrm{M}$ :

$$
\mathrm{M}+\mathrm{X}^{-}=\mathrm{Z}^{-}+\mathrm{P}
$$

Cet ion $\mathrm{Z}^{-}$ainsi produit réagit avec la molécule $\mathrm{U}$ pour donner l'ion instable $\mathrm{Y}^{-}$:

$$
\mathrm{Z}^{-}+\mathrm{U}=\mathrm{Y}^{-}+\mathrm{N}
$$

L'ion $\mathrm{Y}^{-}$peut se décomposer en libérant de l'énergie et redonnant ses composants $\mathrm{X}^{-}$et $\mathrm{U}$ suivant deux mécanismes différents; soit par un mécanisme monomoléculaire:

$$
\mathrm{Y}^{-}=\mathrm{X}^{-}+\mathrm{U}
$$

soit par un mécanisme trimoléculaire:

$$
2 \mathrm{X}^{-}+\mathrm{Y}^{--}=3 \mathrm{X}^{-}+\mathrm{U}
$$

On suppose toutes ces réactions irréversibles.

Le mécanisme complet de la réaction. (A) est ainsi traduit par l'ensemble des quatre réactions:

$$
\begin{array}{r}
2 \mathrm{X}^{-}+\mathrm{Y}^{-} \rightarrow 3 \mathrm{X}^{-}+\mathrm{U}^{-} \\
\mathrm{Y}^{-} \rightarrow \mathrm{X}^{-}+\mathrm{U}^{-} \\
\mathrm{M}+\mathrm{X}^{-} \rightarrow \mathrm{Z}^{-}+\mathrm{P} \\
\mathrm{Z}^{-}+\mathrm{U} \rightarrow \mathrm{Y}^{-}+\mathrm{N}
\end{array}
$$

La somme de ces quatre réactions redonne bien la réaction globale (A). Si l'on admet que les équations chimiques traduisent les mécanismes élémentaires, la cinétique permet d'écrire les vitesses $v$ des réactions. Désignant les concentrations des divers corps par la minuscule correspondant à leur symbole, et les constantes de vitesse par $k_{1}, k_{3}, k_{3}, k_{4}$, on peut alors écrire:

$$
\begin{aligned}
& v_{1}=k_{1} x^{2} y \\
& v_{2}=k_{2} y \\
& v_{3}=k_{3} m x \\
& v_{1}=k_{4} z u
\end{aligned}
$$


Ce système d'équations permet de décrire complètement l'évolution du système.

Il résulte des équations (B) que la quantité totale, soit libre, soit combinée des catalyseurs $\mathrm{X}^{-}$et $\mathrm{U}$, reste constante dans le milieu intérieur isolé, ce qui donne les deux relations: concentration totale de $\mathrm{X}^{-}$:

$$
x+y+z=b=\text { constante }
$$

concentration totale de $\mathrm{U}$ :

$$
y+u=c=\text { constante }
$$

Pour décrire un tel système, il est nécessaire de connaître la concentration $m \mathrm{du}$ corps $\mathrm{M}$ initial, ainsi que les concentrations globales $b$ et $c$ des catalyseurs. Ses propriétés dépendent uniquement de ces trois paramètres lorsque les constantes de vitesse $k$, qui ne dépendent que de la température, sont fixées.

L'étude de l'évolution du système peut se faire par l'analyse mathématique du système d'équations (C). Mais l'essentiel des phénomènes peut être décrit d'une manière simple que nous utiliserons dans cet exposé.

\section{CONSEQUENCES DE L'HYPOTHESE CHIMIQUE}

Pour des raisons de commodité, nous considérons tout d'abord des phénomènes rapides, au cours desquels nous supposons - ce que justifie une étude rigoureuse - que la concentration $y$ ne varie sensiblement pas. Nous montrons que, dans ces conditions, il existe un régime stationnaire dans lequel la réaction (A) se produit avec une vitesse faible et constante.

Nous étudions l'effet d'une variation imposée $\Delta x$ de la concentration $x$. Une telle variation disparaît spontanément si elle est faible, le milieu revenant rapidement à un régime stationnaire. Si elle dépasse un certain seuil, elle déclenche une accélération brusque, mais passagère, de la réaction 1, et le milieu retourne ensuite progressivement à un régime stationnaire. Nous tirerons de ces résultats quelques conséquences qui présentent des analogies formelles avec les propriétés de la cellule excitable. L'hypothèse physique introduite plus loin permettra de transformer ces analogies formelles en analogies physiques précises.

Les ions $\mathrm{X}^{-}$sont produits par les réactions 1 et 2 , avec les vitesses respectives:

$$
\begin{aligned}
& v_{1}=k_{1} y x^{2} \\
& v_{2}=k_{2} y
\end{aligned}
$$

Leur vitesse de formation $v_{F}$ est donc la somme:

$$
\begin{aligned}
& v_{F}=v_{1}+v_{2} \\
& =k_{2} y+k_{1} y x^{2}
\end{aligned}
$$

Ils disparaissent par la réaction 3 avec la vitesse $v_{D}$ :

$$
\begin{aligned}
& v_{D}=v_{3} \\
& =k_{3} m x
\end{aligned}
$$


Leur concentration croît donc avec la vitesse $v_{N}$, qui est la différence de la vitesse de formation $v_{F}$ et de la vitesse de disparition $v_{D}$ :

$$
v_{N}=v_{F}-v_{D}
$$

Portons les vitesses $v_{F}$ et $v_{D}$ en fonction de la concentration $x$ pour une concentration $y$ donnée. Dans la figure 1 , on a supposé que la droite $v_{D}$ coupe la courbe $v_{F}$ : c'est le cas que nous considérerons seul ici. Nous désignerons les abscisses des deux

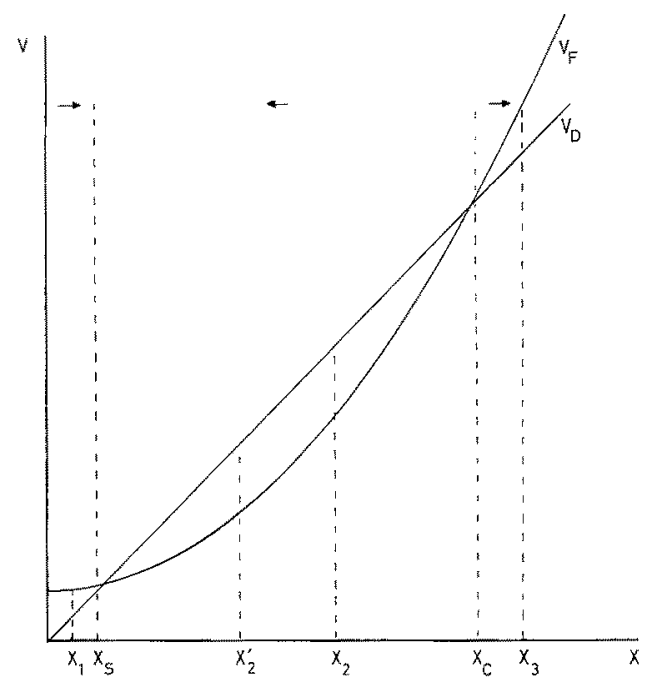

Fig. 1: Effet d'une stimulation brusque

intersections par $x_{3}$ et $x_{c}$. Pour une concentration $x$ donnée, la vitesse nette $v_{N}$ d'accroissement de $x$ est la différence algébrique entre l'ordonnée de la courbe $v_{F}$ et celle de la droite $v_{D}$.

On voit immédiatement sur le diagramme que cette vitesse $v_{N}$ est positive pour $x<x_{s}$, nulle en $x_{s}$, négative pour $x$ compris entre $x_{s}$ et $x_{c}$, nulle en $x_{c}$ et positive pour $x>x_{c}$.

Des deux points $x_{s}$ et $x_{\varepsilon}$, le premier correspond à un régime stationnaire stable de la réaction $(A)$, le second à la limite de stabilité de ce régime.

En effet, les ions $\mathrm{X}^{-}$ayant la concentration $x_{s}$, supposons qu'on impose une variation brusque de concentration $x$ qui déplace $x$ en $x_{1}$. Pour cette valeur de $x$, la vitesse nette $v_{N}$ d'accroissement de $x$ est positive: la concentration crô̂t donc jusqu'à ce qu'elle atteigne $x_{s}$. La vitesse $v_{N}$ s'annule alors, la concentration ne varie plus.

Considérons ensuite une variation de concentration qui déplace $x$ en $x_{2}$. Pour cette valeur de $x$, la vitesse $v_{N}$ est négative. La concentration $x$ doit donc diminuer, et cette diminution s'arrête lorsque le système est revenu en $x_{s}$. Le régime de la réaction (A) est donc stable pour les petites variations de concentration considérées.

Mais supposons maintenant qu'on impose une variation de concentration plus importante qui déplace $x$ en $x_{3}>x_{c}$. Pour cette valeur, la vitesse $v_{N}$ est positive, et 
le système va suivre une évolution dans laquelle la concentration $x$ croît indéfiniment. Le système est excité. Le point critique $x_{c}$ représente donc la limite de stabilité du système pour les variations de la concentration $x$.

Le système revient toujours à son régime stable pour de petites variations de $x$ autour de $x_{s}$, mais s'éloigne de ce régime dès que cette concentration dépasse le seuil $x_{c}$. Dans ce qui suit, nous appellerons «stimulation» un accroissement de concentration $\Delta x$, et $\Delta x_{c}=x_{c}-x_{s}$, «stimulation critique».

\section{EFFET DE STIMULATIONS SUCCESSIVES. SOMMATION}

Le système ayant la concentration $x_{s}$ au temps zéro, accroissons - la brusquement jusqu'en $x_{2}$ par une stimulation $\Delta x_{1}$ (fig. 1). Pour cette valeur de $x$, la vitesse nette $v_{N}$ est négative. La concentration va donc diminuer progressivement. A l'instant $t_{1}$, où la concentration a pris la valeur $x_{2}{ }^{\prime}$, faisons la croître brusquement de $\Delta x_{2}$ en choisissant $\Delta x_{2}<\Delta x_{c}$, c'est à dire insuffisante pour déclencher l'excitation du système lorsqu'il est au repos en $x_{s}$. Partant de $x_{2}^{\prime}$, la concentration croît alors brusquement jusqu'en $x_{3}>x_{c}$, et le système est excité.

Il $\mathrm{y}$ a donc eu sommation des stimulations $\Delta x_{1}$ et $\Delta x_{2}$. Insuffisants pour déclencher seuls la réponse du système, leurs effets s'ajoutent et entraînent l'excitation lorsque les stimulations agissent successivement, le temps $t_{1}$ qui les sépare étant assez petit pour que le système ne soit pas revenu à une valeur de $x$ trop faible lorsque la stimulation $\Delta x_{2}$ est appliquée.

\section{CAS D'UNE STIMULATION DE DUREE, FINIE}

Supposant toujours la concentration $y$ constante, considérons le cas où les ions $\mathrm{X}^{-}$sont apportés dans le milieu par une source qui, en l'absence des réactions (B), accroîtrait la concentration $x$ avec une vitesse:

$$
\frac{d x}{d t}=I_{\mathrm{X}}
$$

Lorsque les réactions ont lieu, la vitesse $I_{X}$ de la source s'ajoute à vitesse de formation $v_{F}$ de la figure 1 . Il revient au même de considérer qu'elle se retranche de la vitesse de disparition $v_{D}$ et la fait passer en $v_{D}{ }^{\prime}, v_{F}$ n'étant pas modifiée. Nous utiliserons cette dernière représentation pour des raisons de commodité (fig. 2).

Supposons tout d'abord la vitesse $I_{X}$ grande devant la vitesse $\omega_{N}$. La vitesse nette d'accroissement de concentration qui était $v_{N}<0$ entre $x_{s}$ et $x_{c}$, devient alors $v_{N}^{\prime}=I_{X}+v_{N}>0$. La concentration croit, et cela d'autant plus rapidement que $I_{X}$ est plus grand. Si $I_{X}$ est assez grand, nous pouvons négliger $v_{N}$ devant $I_{X}$, et considérer la vitesse $v_{N}^{\prime}$ comme sensiblement constante. L'accroissement $\Delta x_{c}=x_{c}-x_{S}$, nécessaire pour exciter le système, est atteint au bout d'un temps $t_{c}$ tel que:

$$
I_{X} t_{c} \simeq x_{c}-x_{s}
$$


ou:

$$
t_{c} \simeq \frac{x_{c}-x_{s}}{I_{X}}
$$

L'excitation est donc obtenue au bout d'un temps $t_{c}$ qui est approximativement inversement proportionnel à $I_{X}$. Dans le cas où la vitesse $v_{N}$ n'est pas négligeable devant $I_{X}$, la relation précédente n'est plus valable, le temps $t_{c}$ devient plus grand que celui qu'elle prévoit. La valeur du produit $I_{X} t_{c}$ nécessaire pour déclencher l'excitation devient alors plus grande que $x_{c}-x_{i}$, une partie des ions

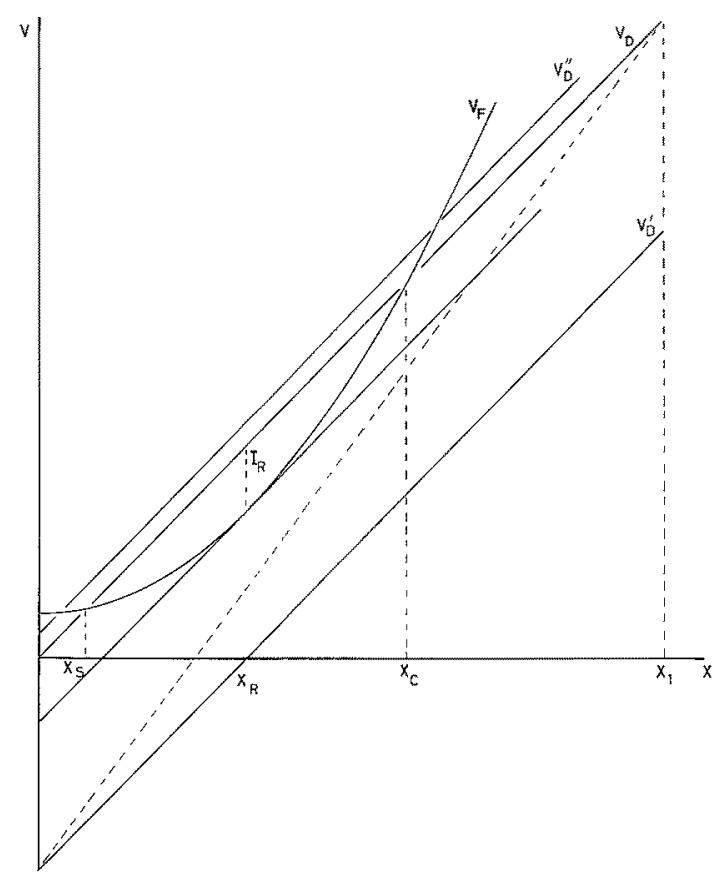

Fig. 2: Effet d'une stimulation progressive

apportés au milieu ayant disparu par la réaction 3. Il est possible d'obtenir mathématiquement une relation rigoureuse que l'on peut comparer aux résultats expérimentaux (Giber T 1953).

Considérons maintenant le cas limite où la vitesse $I_{X}$ est telle que la droite $v_{D R}{ }^{\prime}$ devient tangente à la courbe $v_{F}$ (fig. 2). La concentration passe alors de sa valeur initiale $x_{8}$ aे une valeur $x_{R}$ où la vitesse $v_{N}^{\prime}$ est nulle; la concentration ne varie plus, et l'excitation du système n'est pas déclenchée. La vitesse $I_{R}$ qui correspond à ce cas est donc une limite inférieure des vitesses susceptibles de produire une excitation du système.

On remarque entre la vitesse $I_{R}$ ainsi définie et la notion de rhéobase une analogie formelle que préciserait l'étude de l'effet de la variation de $y$ et de la relation entre $I_{X}$ et l'intensité du courant électrique de stimulation. 
Si l'on enlève des ions $\mathrm{X}^{-\prime}$ au milieu au lieu d'en ajouter, la vitesse de cette «source négative» fait passer la droite $v_{D}$ en $v_{D}{ }^{\prime \prime}$ et l'excitation n'est pas déclenchée.

\section{EFFET D'UNE ADDITION LENTE D'IONS $\mathrm{X}-$}

Dans ce qui précède, on a supposé que l'addition d'ions $\mathrm{X}^{-}$était rapide: les réactions 3 et 4 n'avaient pas le temps d'intervenir, la concentration des ions $\mathrm{Y}^{-}$ne yariait pas, et tous les ions $\mathrm{X}^{-}$ajoutés contribuaient à élever la concentration $x$.

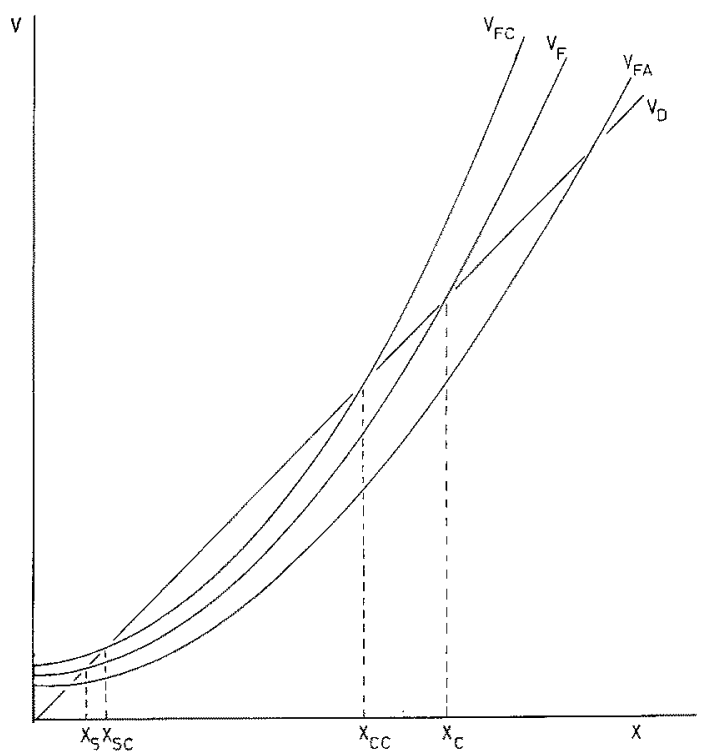

Fig. 3: Effer de l'accommodation

Mais supposons maintenant que l'addition d'ions $\mathrm{X}^{-}$soit assez lente pour permettre aux réactions 3 et 4 d'intervenir; les ions $X^{-}$ajoutés se transforment alors en ions $\mathrm{Z}^{-}$et $\mathrm{Y}^{-}$, leur concentration $x$ ne varie que très peu, et l'on atteint un nouveau régime stationnaire voisin du régime initial.

L'étude rigoureuse montre que, pour un choix convenable des concentrations $b, c$ et $m$, la concentration $y$ est beaucoup plus grande que $x$ dans le régime stationnaire initial. Il résulte de là que, à une variation relative importante de la concentration $x$ correspond une variation relative faible de la concentration $y$.

Les ordonnées de la courbe $v_{F}$ étant proportionnelles à $y$, l'addition lente d'ions $\mathrm{X}^{-}$a alors pour effet de relever progressivement la courbe $v_{F}$, qui devient ainsi telle que $v_{F C}$ (fig. 3), la droite $v_{D}$ n'étant pas modifiée.

Il en est ainsi pour l'addition lente d'une quantité d'ions $\mathrm{X}^{-}$qui, effectuée brusquement, porterait la concentration $x$ à droite du seuil $x_{c}$ et déclencherait l'excitation: il y a accommodation, et l'excitation n'est pas déclenchée. Pour la 
nouvelle courbe $v_{F C}$, la différence $x_{c c}-x_{s c}$ est plus faible que la différence $x_{c}-x_{s}$ relative à la courbe $v_{F}$ : la stimulation critique est donc diminuée. En outre, la vitesse limite $I_{R}$ considérée plus haut, correspondant à la rhéobase, est également diminuée quand on passe de $v_{F} \grave{a} v_{F C}$, comme on s'en rend compte aisément sur la figure.

La soustraction d'ions $X^{-}$au milieu produit des effets inverses: elle fait passer la courbe $v_{F}$ en $v_{F A}$ : les conclusions relatives à cette courbe sont évidemment inverses des précédentes: il y a accroissement simultané de la stimulation critique et de la rhéobase. Ces résultats seront complétés plus loin.

\section{EVOLUTION DU SYSTEME APRĖS EXCITATION}

Dans le cas où la concentration $x$ a dépassé le point $x_{c}$ de la figure 1 , si les variations de $y$ restaient négligeables, la concentration $x$ continuerait à croître indéfiniment.

En fait, il résulte des réactions 1 et 2 que, pour $x>x_{c}$ et tant que les réactions 3 et 4 sont négligeables, l'accroissement de $x$ est accompagné d'une diminution corrélative de $y$. Lorsque la concentration $y$ est devenue suffisamment petite, les vitesses $v_{1}$ et $v_{2}$ deviennent très faibles, et la vitesse $v_{F}$ de formation de $\mathrm{X}^{-}$négligeable. Mais alors, la concentration des ions $\mathrm{X}^{-}$est importante, la réaction 3 devient

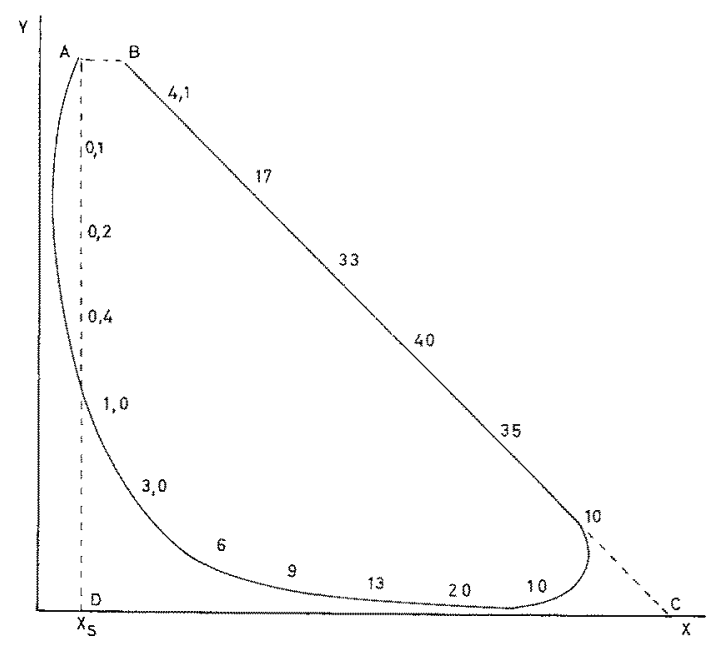

Fig. 4: Evolution de la composition au cours de l'excitation. Les nombres portés sur la courbe sont proportionnels à la vitesse de déplacement du point représentatif

rapide, et synthétise les ions $Z^{-}$en faisant disparaître les ions $\mathrm{X}^{-}$. Enfin, lorsque la concentration initiale en $\mathrm{Y}^{-}$, ramenant le système au régime stationnaire initial.

Si l'on porte la concentration $y$ en ordonnées en fonction de la concentration $x$ prise en abscisses, on peut représenter la réponse du système à une excitation par le 
schéma de la figure 4. Le point A représente le régime stationnaire initial. L'accroissement $\Delta x$ est celui qui fait passer la concentration de $x_{s}$ à une valeur $x_{3}>x_{c}$, et porte ainsi le système au point $B$, où se produit l'excitation. Sur le segment $B C$ a lieu la réaction 1 , rapide, les réactions 3 et 4 restant négligeables. Elle porte le système $\mathrm{au}$ point $\mathrm{C}$ où $y$ est sensiblement nul, et où $x$ prend sa valeur maximale. La concentration $x$ étant alors élevée, la réaction 3 fait passer le point représentatif en $D$, par disparition des ions $\mathrm{X}^{-}$et synthèse des ions $\mathrm{Z}^{-}$, et la réaction 4 le ramène finalement au point initial A par synthèse de $\mathrm{Y}^{-}$à partir de $\mathrm{Z}^{-}$.

Cette figure est schématique. L'intégration numérique du système d'équations (C) montre que, pour des valeurs convenablement choisies des concentrations $b, c$ et $m$, on obtient en fait une évolution dont l'allure est donnée qualitativement par la courbe en trait plein. A la place des angles vifs, cette courbe comporte des parties arrondies. Au voisinage du point $\mathrm{C}$, cette allure traduit le fait que la réaction 3 commence à prendre de l'importance avant que les réactions 1 et 2 soient terminées. De même, au voisinage du point $D$, la réaction 4 commence avant que la réaction 3 soit terminée. La concentration des ions $Z^{-}, z=b-x-y$, est faible dans le régime stationnaire et sur le segment $B C$. Elle ne prend une valeur importante qu'au voisinage du point $\mathrm{D}$.

\section{PÉRIODES REFRACTAIRES}

Au cours de l'évolution du système, la concentration $y$ varie. Il en est de même des vitesses $v_{1}$ et $v_{2}$ qui lui sont proportionelles, donc aussi de la ritesse de formation $v_{F}$. Dans le diagramme de la figure 1 , la courbe $v_{F}$ se déplace verticalement quand la concentration $y$ varie.

Si l'on applique une excitation $\Delta x$ pendant que les système parcourt la partie $\mathrm{BC}$ de la figure 4, elle ne fait qu'ajouter à la vitesse de formation de $\mathrm{X}^{-}$, déjà grande, et ne produit pas d'effet sensible. En outre, la diminution de $y$ entraîne un accroissement de la stimulation critique. Pendant le parcours du segment $C D$, la concentration de $\mathrm{Y}^{-}$reste négligeable, la vitesse de formation $v_{F}$ sensiblement nulle, la courbe $v_{E}$ de la figure 1 reste voisine des abscisses, l'effet d'une excitation $\Delta x$ est nul (période réfractaire absolue).

Le long du segment D A de la figure 4, $y$ croissant, la courbe $v_{F}$ s'élève progressivement. La distance entre les intersections de la courbe $v_{F}$ et de la droite $v_{D}$ diminue corrélativement au fur et à mesure que $y$ croît. La stimulation critique $\Delta x_{c}$, capable de produire une réponse du système, diminue donc progressivement, et la sensibilité du système croît en même temps que $y$ (période réfractaire relative). Cette croissance continue jusqu'à ce que le point A soit atteint.

Les analogies formelles que l'on peut constater entre ces résultats et ceux de l'observation physiologique seront précisées par l'hypothèse physique que nous considérerons maintenant, et qui permettra de relier les variations de concentration $\Delta x$ à la stimulation électrique qui les produit. 


\section{HYPOTHESE PHYSIQUE}

On suppose les ions $\mathrm{Y}^{-}, \mathrm{Z}^{-}$et les molécules $U$ fixes, non diffusibles et localisés dans le milieu intérieur où ont lieu les réactions considérées précédemment. Les ions $\mathrm{X}^{-}$- et les cations $\mathrm{C}^{+}$nécessaires à la neutralité électrique - sont diffusibles et peuvent migrer vers le milieu extérieur, dans lequel baigne le premier. La surface $S$ de séparation de ces deux milieux est alors analogue à surface d'un échangeur d'ions plongé dans une solution (Hellferich 1963).

Les réactions chimiques considérées ont alors lieu uniquement dans le milieu intérieur, et les deux milieux ne réagissent l'un sur l'autre que par l'intermédiaire de la migration des ions $\mathrm{X}^{-}$et $\mathrm{C}^{+}$à travers la surface de séparation. Il reste à déduire les conséquences de cette hypothèse.

\section{CONSÉQUENCES DE L'HYPOTHESE PHYSIQUE}

Le milieu intérieur étant électriquement neutre, la concentration w des cations $\mathrm{C}^{+}$est égale à la concentration totale des anions:

$$
\begin{aligned}
& w=x+y+z \\
& w=b
\end{aligned}
$$

Le paramètre $b$ est donc égal à la concentration des cations dans le milieu intérieur (si l'on néglige tout ion différent de $\mathrm{X}^{-}, \mathrm{Y}^{-}, \mathrm{Z}^{-}$et $\mathrm{C}^{+}$). Dans l'état stationnaire, il s'etablit un équilibre de Donnan entre les deux milieux.

On sait (Hellferich 1963) que, dans un tel équilibre, et en assimilant les activités aux concentrations, il y a égalité des produits des concentrations des ions diffusibles des deux côtés de la surface de séparation. Si l'on désigne par $x^{\prime}$ et $w^{\prime}$ les concentrations respectives des anions $\mathrm{X}^{-}$et des cations $\mathrm{C}^{+}$dans le milieu extérieur:

$$
x w=x^{\prime} w^{\prime}
$$

$\mathrm{Si}$ nous supposons que le milieu extérieur contient uniquement les ions $\mathrm{X}^{-}$et $\mathrm{C}^{+}$, les concentrations de ces ions sont égales et l'on a:

ou, d'après (5):

$$
x w=x^{2}
$$

$$
x(x+y+z)=x^{2}
$$

Pour fixer les idées, supposons, en prenant des unités arbitraires:

on trouve alors:

$$
x^{\prime}=20 \quad y+z=200
$$

$$
x=2 \quad w=202
$$

La concentration du milieu intérieur en ions $X^{-}$est le $\frac{1}{10}$ de celle du milieu extérieur, la concentration en ions $\mathrm{C}^{+} 10$ fois plus grande que celle du milieu extérieur. 
On sait, en outre, qu'il existe, à l'équilibre, une différence de potentiel E entre le milieu extérieur et le milieu intérieur (Hellferich 1963), le potentiel de Donnan qui est exprimé à $25^{\circ} \mathrm{C}$ par la relation ( $\mathrm{E}$ en millivolts):

Dans l'exemple considéré:

$$
\mathrm{E}=59 \log \frac{x^{\prime}}{x}
$$

$$
\mathrm{E}=59 \mathrm{mV}
$$

Lorsque la relation (7) n'est pas satisfaite, l'équilibre est rompu.

Si:

$$
x w<x^{\prime} w^{\prime}
$$

les ions du milieu extérieur migrent vers l'intérieur, tendant à rétablir l'équilibre. L'effet de cette migration est assimilable à celui d'une source d'ions $\mathrm{X}^{-}$telle que celle qui a été considérée, et peut, par suite, provoquer l'excitation. Toute modification. chimique qui accroît le produit des activités des ions $\mathrm{C}^{+}$et $\mathrm{X}^{-}$a évidemment le même effet (excitation chimique).

Une dilution du milieu extérieur primitivement à l'équilibre entraîne:

$$
x w>x^{\prime} w^{\prime}
$$

Les ions $\mathrm{X}^{-}$migrent alors vers l'extérieur, la concentration $x$ diminue, ce qui ne peut pas produire d'excitation d'après les résultats du cas d'une stimulation de durée finie.

\section{MIGRATION DES IONS SOUS L'EFFET D'UNE STIMULATION ÉLECTRIQUE}

Supposons que on fasse passer un courant électrique d'intensité I de l'intérieur vers l'extérieur. Si l'on considère le cas où $x^{\prime}=w^{\prime}$ et en supposant les mobilités des ions $\mathrm{X}^{-}$et $\mathrm{C}^{+}$voisines, le courant est transporté sensiblement par moitié par chacun de ces ions dans le milieu extérieur. Mais, dans le milieu intérieur, $x \ll w$ et les ions $\mathrm{Y}^{-}$et $\mathrm{Z}^{-}$, non diffusibles, ne participent pas à la conduction: le courant est donc transporté uniquement par les ions $\mathrm{C}^{+}$. Ne pouvant traverser la surface, les ions $\mathrm{X}^{-}$ s'accumulent à son voisinage immédiat, retenant un nombre égal d'ions $\mathrm{C}^{+}$(RASHEvsky 1950). Cet accroissement de concentration provoque un déséquilibre qui, entraîne la migration des ions $\mathrm{X}^{-}$vers l'intérieur, ce qui est analogue à la source d'ions $\mathrm{I}_{\mathrm{X}}$, dont les résultats s'appliquent qualitativement (excitation électrique). On obtient donc l'excitation si l'intensité du courant et le temps de stimulation sont suffisants pour que la concentration $x_{C}$ soit atteinte. Mais si l'intensité est inférieure à une certaine limite, la vitesse $I_{R}$ n'est pas atteinte, et il n'y a plus d'excitation.

Supposons qu'un tel courant subliminaire persiste pendant un temps assez long, et considérons ce qui se passe lorsque un régime stationnaire de migration est atteint. Le passage du courant d'une électrode à l'autre a lieu successivement à travers le milieu extérieur, puis le milieu intérieur, et de nouveau à travers le milieu extérieur.

Ce phénomène est analogue au passage du courant à travers une membrane ionique. Le passage du courant en régime permanent entraîne une redistribution des 
ions $\mathrm{X}^{-}$telle que celle qui est représentée dans la figure 5, où l'on a porté la concentration des ions $\mathrm{X}^{-}$en ordonnées en fonction du point considéré. La courbe en trait plein représente la distribution des ions $\mathrm{X}^{-}$en absence de courant, la courbe en pointillé ce qu'elle devient sous l'effet du courant.

Du côté de la cathode C, la concentration dans le milieu extérieur est accrue au voisinage de la membrane: il en est de même de la concentration dans le milieu intérieur par suite de l'équilibre de Donnan. On observe l'effet inverse du côté de l'anode. Les réactions chimiques $(B)$ qui ont lieu dans le milieu intérieur ont alors pour conséquence

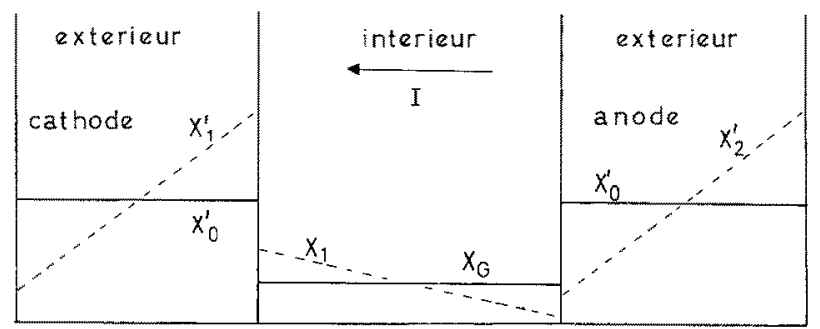

Fig. 5: Modification des concentrations pendant le passage du courant dans un échangeur d'ions

l'accroissement de la concentration y du côté de la cathode, sa diminution du côté de l'anode. De chaque côté, un nouveau régime stationnaire est atteint. Du côté de la cathode, la courbe $v_{F}$ est remplacée par la courbe $v_{F C}$ (fig. 3), qui coupe la droite $v_{D}$ en $x_{s c}$ et $x_{c c}$. La différence $x_{c c}-x_{s c}$ est plus petite que la différence $x_{c}-s_{s}$ des points d'intersection avec $v_{F}$, dans l'absence de courant. La stimulation critique est donc diminuée du côté de la cathode, ainsi que la rhéobase.

Du côté de l'anode, la courbe $v_{F}$ est remplacée par $v_{F A}$ qui montre que la stimulation critique est augmentée et la rhéobase également. On remarque l'analogie avec les caractères de l'électrotonus. Dans le cas de l'anélectrotonus, on voit sur la figure 5 que la concentration des ions $\mathrm{X}^{-}$dans le milieu extérieur est abaissée. A l'ouverture du courant, les ions du milieu extérieur se redistribuent très rapidement, leur concentration reprend la valeur $x_{0}{ }^{\prime}$ de repos, ce qui entraîne un accroissement brusque de la concentration $x^{\prime}$ à l'extérieur, dont la variation est la même qu'elle avait été du côté de la cathode lors de la fermeture. Mais, la stimulation critique étant plus élevée du côté de l'anode, un courant plus intense est nécessaire pour l'excitation d'ouverture, à l'anode, que pour l'excitation de fermeture, à la cathode.

\section{EVOLUTION DU POTENTIEL D'ACTION}

Dans le régime stationnaire le potentiel de Donnan résulte de la présence des ions non diffusibles $\mathrm{Y}^{-}$et $\mathrm{Z}^{-}$dans le milieu intérieur. Pendant la phase $\mathrm{BC}$ de la réponse (fig. 4), les ions $\mathrm{Y}^{-}$disparaissent et font place à des ions $\mathrm{X}^{-}$diffusibles. Le potentiel de Donnan diminue donc corrélativement. La concentration des ions $\mathrm{X}^{-}$ croît jusqu'au point $\mathrm{C}$, où les ions $\mathrm{Y}^{-}$ont disparu, et où il ne reste que des ions $\mathrm{Z}^{-}$, dont 
la concentration est faible. A ce moment, le milieu intérieur est devenu sensiblement un électrolyte ordinaire constitué presque exclusivement par des ions mobiles $\mathrm{X}^{-}$et $\mathrm{C}^{+}$, à des concentrations voisines et sensiblement égales à la concentration initiale des ions $\mathrm{C}^{+}$.

Dans l'exemple que nous avons choisi, dans le milieu intérieur:

et, dans le milieu extérieur:

$$
x=w=200
$$

$$
x^{\prime}=w^{\prime}=200
$$

De là résultent les conséquences suivantes: (1) Le milieu intérieur devenant une solution électrolytique ordinaire, la surface de séparation perd ses propriétés sélectives et devient perméable à tous les ions («effondrement» de la membrane). (2) Le potentiel de DonNan disparaît pour faire place à un potentiel de concentration et de diffusion.

Sur le segment $C D$, les ions mobiles $\mathrm{X}^{-}$disparaissent au profit des ions fixes $Z^{-}$, la conductivité diminue et le potentiel initial se rétablit progressivement. Enfin, sur le segment DA, la concentration en ions $\mathrm{X}^{-}$passe par un minimum, et il en est de même du potentiel.

La relation (10) qui exprime le potentiel dans le cas de l'équilibre de Donnan est, approximativement, valable dans le cas de la pile de concentration, avec des électrodes convenables et en supposant voisins les nombres de transport des deux ions. On obtient donc une idée qualitative $d u$ potentiel d'action en porrant $\log \frac{x}{x_{\mathbb{s}}}$ en fonction du temps $t$ au cours de l'évolution. L'allure générale des courbes obtenues est celle de la figure $6 a$ pour un choix convenable des paramètres $b, c, m$ : elles comportent un

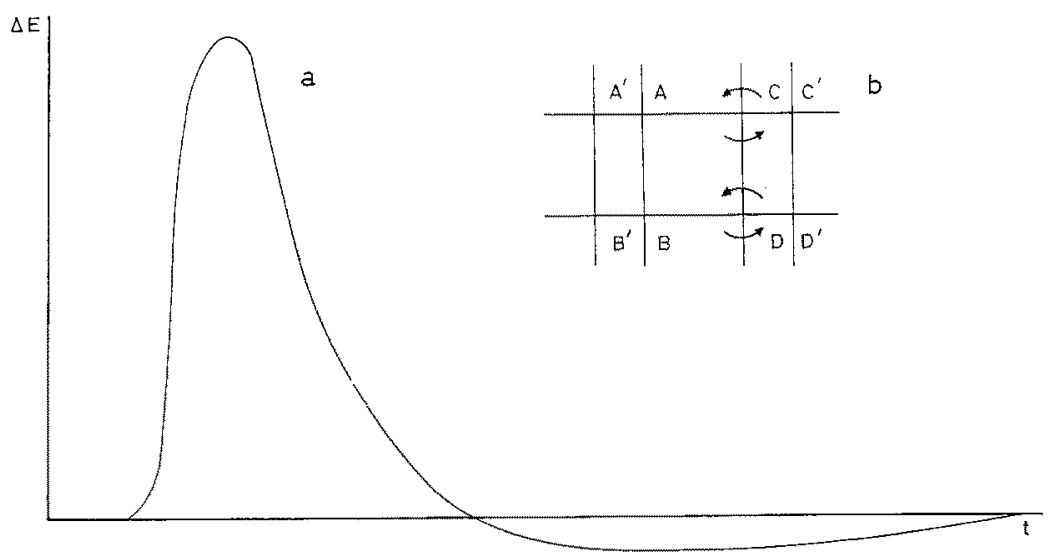

Fig. 6: (a) Allure du potentiel d'action, (b) Mécanisme de la propagation

maximum positif aigu suivi d'un minimum négatif moins marqué. Mais la ressemblance d'une telle courbe avec celles que l'on obtient expérimentalement doit être considérée comme formelle, car le modèle ne comporte qu'un seul électrolyte diffusible $\mathrm{X}^{-} \mathrm{C}^{+}$, 
et les phénomènes qui ont lieu dans la cellule vivante sont certainement plus complexes que ceux qu'il présente.

\section{PROPAGATION}

Il résulte des propriétés précédentes que, dans le cas où la surface de séparation $S$ est cylindrique, l'excitation développée en un point se propage parallèlement à l'axe, même si elle reste limitée à une faible épaisseur du milieu intérieur.

En effet, l'excitation déclenchée entre les plans $\mathrm{AB}$ et $\mathrm{CD}$ (fig. $6 \mathrm{~b}$ ) provoque un abaissement du potentiel extérieur, d'où une différence de potentiel entre la partie de la surface contenue entre $C D$ et $C^{\prime} D^{\prime}$ et celle contenue entre $A B$ et $C D$. II en résulte un courant $I$ qui passe de la surface au repos vers la surface excitée, et déclenche l'excitation dans la partie au repos. L'excitation se propage ainsi vers la droite. Elle se propage de même vers la gauche, sauf si la partie du système contenue entre $A^{\prime} B^{\prime}$ et $\mathrm{AB}$ se trouve dans la période réfractaire.

\section{PRINCIPE DE L'ÉTUDE RIGOUREUSE DE LA CINETIIQUE DE LA REACTION A}

Ces considérations montrent que de nombreux résultats peuvent être déduits par des raisonnements qualitatifs. Toutefois, nous signalons la possibilité d'une étude plus rigoureuse, qui serait utile pour l'identification des corps $\mathrm{X}^{-}, \mathrm{Y}^{-}, \mathrm{Z}^{-}$et $\mathrm{U}$, et nécessaire pour l'application de ces idées à l'étude de certains cas importants, tels que celui de l'automatisme. En outre, une telle étude permet de justifier les ordres de grandeur admis pour les diverses concentrations.

La cinétique permet d'écrire les vitesses de formation de chacun des corps $\mathrm{X}^{-}, \mathrm{Y}^{-}$, $\mathrm{Z}^{-}$, $\mathrm{U}$ en fonction des diverses concentrations (équations $\mathrm{C}$ ). $\mathrm{L}^{\prime}$ 'ion $\mathrm{X}^{-}$se forme par les réactions (11) et (12) et disparaît par la réaction (13). Sa vitesse nette de formation vaut ainsi:

$$
\begin{aligned}
\frac{d x}{d t} & =v_{1}+v_{2}-v_{3} \\
& =k_{1} x^{2} y+k_{2} y-k_{3} m x
\end{aligned}
$$

L'ion $\mathrm{Y}^{-}$disparaît par les réactions (11) et (12), et apparaît dans la réaction (14). D'où:

$$
\begin{aligned}
\frac{d y}{d t} & =-v_{1}-v_{2}+v_{4} \\
& =-k_{1} x^{2}-k_{2} y+k_{4} z u
\end{aligned}
$$

L'ion $Z^{-}$apparaît par la réaction (13) et disparaît par la réaction (14):

$$
\begin{aligned}
\frac{d z}{d t} & =v_{3}-v_{4} \\
& =k_{3} m x-k_{4} z u
\end{aligned}
$$


Enfin, la molécule U apparaît dans les réactions (11) et (12) et disparaît par la réaction (14):

$$
\begin{aligned}
\frac{d u}{d t} & =v_{1}+v_{2}-v_{4} \\
& =k_{1} x^{2} y+k_{2} y-k_{4} z u
\end{aligned}
$$

On voit immédiatement que:

D'où:

$$
\frac{d x}{d t}+\frac{d y}{d t}+\frac{d z}{d t}=0
$$

De même:

$$
x+y+z=\text { constante }=b
$$

D’où:

$$
\frac{d y}{d t}+\frac{d u}{d t}=0
$$

$$
y+u=\text { constante }=c
$$

Eliminant $z$ et $u$ des équations (11) et (12) grâce aux relations (15) et (16), il vient:

$$
\begin{aligned}
& \frac{d x}{d t}=k_{1} x^{2} y+k_{2} y-k_{3} m x \\
& \frac{d y}{d t}=-k_{1} x^{2} y-k_{2} y+k_{4}(c-y)(b-x-y)
\end{aligned}
$$

Les équations (17) et (18) contiennent 7 paramètres: $k_{1}, k_{2}, k_{3}, k_{4}, m, b, c$. Leur étude est simplifiée si on les écrit sous forme réduite en posant:

$$
\begin{gathered}
x=\xi\left(\frac{k_{2}}{k_{1}}\right)^{\frac{1}{2}} \quad y=\eta\left(\frac{k_{2}}{k_{1}}\right)^{\frac{1}{2}} \quad b=\beta\left(\frac{k_{2}}{k_{1}}\right)^{\frac{1}{2}} \quad c=\gamma\left(\frac{k_{2}}{k_{1}}\right)^{\frac{1}{2}} \\
m=\mu \frac{k_{2}}{k_{3}} \quad t=\frac{\Theta}{k_{2}} \quad \frac{k_{4}}{\left(k_{1} k_{2}\right)^{1 / 2}}=a
\end{gathered}
$$

Elles prennent alors la forme:

$$
\begin{aligned}
& \frac{d \xi}{d \Theta}=\eta\left(\xi^{2}+1\right)-\mu \xi \\
& \frac{d \eta}{d \Theta}=-\eta\left(\xi^{2}+1\right)+\alpha(\gamma-\eta)(\beta-\xi-\eta)
\end{aligned}
$$

où il ne subsiste que les 4 paramètres sans dimensions $\alpha, \beta, \gamma, \mu$, dont le premier dépend seulement de la température, et les trois autres sont proportionnels aux concentrations $b, c, m$.

Un système non linéaire tel que (19), de la forme générale:

$$
\frac{d \xi}{d \Theta}=P(\xi, \eta) \quad \frac{d \eta}{d \Theta}=\mathrm{Q}(\xi, \eta)
$$

peut être résolu par le calcul analogique. Toutefois, l'étude des points singuliers (CUNNINGHAM 1963) permet de prévoir la nature des solutions. Les points singuliers ont des coordonnées $\xi_{0}$, no telles que:

$$
p\left(\xi_{0}, \eta_{0}\right)=0 \quad \mathrm{Q}\left(\xi_{0}, \eta_{0}\right)=0
$$


Le comportement des solutions au voisinage d'un point singulier est régi par le système linéaire tangent:

$$
\frac{d u}{d \Theta}=a_{1} u+b_{1} v \quad \frac{d v}{d \Theta}=a_{2} u+b_{2} v
$$

obtenu en substituant:

$$
\xi=\xi_{0}+u \quad \eta=\eta_{0}+v
$$

dans les équations (20), et en négligeant les termes dont le degré en $u$ et $v$ est supérieur au premier.

Le système (22) admet des solutions de la forme:

$$
\begin{aligned}
& u=u_{0} \mathrm{e}^{r \Theta} \\
& v=v_{0} \mathrm{e}^{r \Theta}
\end{aligned}
$$

où $r$ est solution de l'équation du second degré:

$$
r^{2}-\left(a_{1}+b_{2}\right) r+a_{1} b_{2}-a_{2} b_{1}=0
$$

Le comportement des solutions au voisinage du point singulier dépend de la nature des racines de l'équation (25), qui est déterminée par les signes de trois expressions: la somme $\Sigma$ des racines:

leur produit $\pi$ :

$$
\Sigma=r_{1}+r_{2}=a_{1}+b_{2}
$$

$$
\pi=r_{1} r_{2}=a_{1} b_{2}-a_{2} b_{1}
$$

et le discriminant $\Delta$ de l'équation:

$$
\Delta=\left(a_{1}-b_{2}\right)^{2}+4 a_{2} b_{1}
$$

La discussion (Cunningham 1963) se résume dans le tableau 1.

Tableau 1

Nature des racines de l'equation (25)

\begin{tabular}{|c|}
$\Delta>0$ racines réelles \\
$\pi>0$ de même signe \\
$\qquad$\begin{tabular}{c|}
$\sum \geqslant 0$ positives = noeud répulsif \\
$\pi<0$ de signes contrivaires = col attractif
\end{tabular} \\
\hline$\Delta<0$ racines imaginaires \\
$\sum \geq 0$ partie réelle positive = foyer répulsif \\
$\Sigma<0$ partie réelle négative = foyer attractif
\end{tabular}

Ce mode d'étude donne des résultats facilement utilisables si on l'applique au système simplifié (à 3 paramètres):

$\frac{d \xi}{d \Theta}=\eta\left(1+\xi^{2}\right)-\mu \xi \quad \frac{d \eta}{d \Theta}=-\eta\left(1+\xi^{2}\right)+\lambda(\beta-\xi-\eta)$

que l'on obtient à partir de (19), si l'on suppose:

$$
\eta \ll \gamma \quad(y \ll c)
$$


et sil'on pose:

$$
a \gamma=\lambda
$$

La position des points singuliers (26) est déterminée par le système:

$$
\eta=\frac{\mu \xi}{1+\xi^{2}} \quad \eta=\beta-\frac{\mu+\lambda}{\lambda} \xi
$$

qui peut admettre 1 ou 3 racines convenant au problème. On introduit la variable auxiliaire:

$$
\mathrm{X}=1+\xi^{2}
$$

La nature des points singuliers dépend des signes de:

$$
\begin{aligned}
& \Sigma=-\frac{2 \mu}{\mathrm{X}}+(\mu-\lambda)-\mathrm{X} \\
& \pi=(\mu+\lambda)\left[\mathrm{X}-\frac{\mu \lambda}{\mu+\lambda}+\frac{2}{\mathrm{X}} \frac{\mu \lambda}{\mu+\lambda}\right] \\
& \Delta=\left[\mathrm{X}+\mu+\lambda-\frac{2 \mu}{\mathrm{X}}\right]^{2}-4(2 \mu+\lambda) \mathrm{X}+8 \mu
\end{aligned}
$$

On peut obtenir des solutions très diverses suivant les valeurs attribuées aux paramètres. Si l'on choisit:

$$
\lambda>8 \quad \frac{\mu \lambda}{\mu+\lambda}>8 \quad \frac{\mu \lambda}{\mu+\lambda}>\frac{\lambda}{2}\left[1+\sqrt{\frac{2}{\lambda+2}}\right]
$$

$\Sigma$, $\pi$ et $A$ changent de signe dans l' intervalle $0<\xi<\infty$. Pour des valeurs de $\lambda$ et de $\mu$ qui satisfont assez largement à ces conditions, on peut trouver des valeurs de $\beta$ telles que le système obéit à la loi du tout ou rien.

$$
\text { Exemple: } \quad \lambda=20 \quad \mu=80
$$

Suivant la valeur de $\beta$, on obtient les résultats suivants:

$\beta<38,5$ : il existe un seul point singulier stable (noeud ou foyer) pour lequel $\eta_{0} \gg \xi_{0}$. Ecarté de ce régime stable, le système $y$ revient soit directement, soit après quelques oscillations décroissantes. Il est inexcitable.

$38,5<\beta<45,3$ : il existe 3 points singuliers $A$, B et $C$, tels que $\xi_{\mathrm{A}}<\xi_{\mathrm{B}}<\xi_{\mathrm{C}}$. A est un point stable (noeud ou foyer) pour lequel $\eta_{0} \gg \xi_{0}$. B est instable (col). C est instable (noeud répulsif pour $38,5<\beta<38,7$, et foyer répulsif pour $38,7<\beta<45,3$ ). Dans ce dernier cas, le comportement du système est celui qui a été analysé en détail dans ce mémoire. Il satisfait à la loi du tout ou rien.

$45,3<\beta<48$ : un seul point singulier instable (foyer répulsif). Le système évolue indéfiniment suivant un cycle limite, auquel correspondent des oscillations de concentration régulièrement entretenues.

$\beta>48$; un seul point singulier stable (foyer ou noeud) pour lequel $\xi_{0} \gg \eta_{0}$.

Cet exemple montre la possibilité de préciser le lien entre les facteurs chimiques et le comportement physiologique. Ainsi, on constate, d'une part, que des variations de composition accompagnent l'excitation, de l'autre, que des variations de composition imposées peuvent faire passer un système oscillant à plusieurs états différents où les oscillations s'arrêtent: (1) un état absolument stable où la valeur de $x$ est élevée, celle 
de $y$ faible, et le système totalement inexcitable. (2) un état de stabilité relative, où les oscillations s'arrêtent, le système restant excitable. (3) un second état absolument stable où la valeur de $x$ est faible, celle de $y$ relativement élevée, et le système totalement inexcitable.

\section{DISCUSSION}

Le modèle proposé est évidemment trop simple pour représenter tous les phénomènes observés. En particulier, il ne fait intervenir qu'un seul cation, dont le rôle est analogue à celui de l'ion potassium de la cellule, alors que l'importance du comportement de l'ion sodium a été mise en évidence expérimentalement depuis longtemps (Hodgkin 1963). L'introduction de plusieurs électrolytes diffusibles au lieu des seuls ions $\mathrm{X}^{-}$et $\mathrm{C}^{+}$entraînerait des modifications notamment dans la courbe $6 \mathrm{a}$ du potentiel d'action.

Les développements ultérieurs devront donc faire appel à des hypothèses supplémentaires. Par exemple, on peut supposer que la pompe à sodium (HoDGKIN 1963) est lièe à la réaction $\mathrm{B} 2$, de vitesse:

$$
v_{2}=k_{2} y
$$

En régime stationnaire, la concentration $y$ est élevée, la vitesse $v_{2}$ aussi, la pompe chasse les ions sodium vers le milieu extérieur jusqu'à ce que soit atteinte une différence de concentration suffisante pour que la migration inverse par diffusion compense le débit de la pompe.

Lors de l'excitation, la concentration $y$ tombe à une valeur très faible, le débit de la pompe à sodium également, et la diffusion persiste seule, produisant la migration des ions sodium vers l'intérieur.

Il $y$ aurait lieu également de faire intervenir la couche double ionique, liée à la polarisation, qui existe à la surface de séparation des deux milieux. Son comportement est qualitativement analogue à celui que nous avons considéré, et l'importance relative des deux mécanismes ne pourrait être mise en évidence que par une étude quantitative. De même, les propriétés particulières de la membrane et de l'axoplasme, ainsi que des autres structures qui ont été observées dans la cellule, n'ont pas été explicitées: toute la cellule a été représentée par un seul «milieu intérieur» simple, que l'on peut assimiler provisoirement à la membrane. Il est certain que des phénomènes sont ignorés dans une telle représentation, qui devra être perfectionnée ultérieurement. Enfin, les quatre réactions chimiques postulées ne sauraient être qu'une schématisation très simplifiée des réactions réelles. $\mathrm{La}$ clef de la correspondance avec les phénomènes réels pourrait résider dans le comportement de l'ion $\mathrm{Y}^{-}$, qui serait à comparer à celui des corps présents dans la cellule, tels, par exemple, que l'ATP. Mais de tels problèmes sortent du cadre de cet exposé, et nous ne les envisagerons pas ici.

Le modèle présenté ici ne peut ainsi être considéré que comme une première esquisse. Une comparaison beaucoup plus détaillée avec l'expérience est nécessaire pour décider s'il doit être rejeté, ou, au contraire, conservé et perfectionné. 


\section{RÉSUMÉ}

1. Un modèle simple de cellule excitable est décrit, constitué par un échangeur d'ions qui est le siège d'une réaction chimique déterminée.

2. Les propriétés essentielles de ce modèle sont déduites. Il est montré qu'il simule certains phénomènes physiologiques connus: stimulation chimique et électrique, loi du tout ou rien, accommodation, sommation des stimulations, relation intensitédurée de stimulation, rhéobase, périodes réfractaires, électrotonus, potentiels d'action, propagation de l'excitation.

3. L'étude mathématique explicite les relations entre la composition et les propriétés du système. A des compositions différentes d'un même système correspondent la stabilité absolue, la stabilité relative (loi du tout ou rien), l'instabilité essentielle (oscillations).

4. En particulier, dans le cas d'un système oscillant, des variations convenables de la composition le font passer à divers états stables de propriétés différentes.

5. L'intérêt d'un tel modèle abstrait ne peut se manifester que dans une confrontation approfondie avec l'expérience, qui n'est qu'amorcée ici.

\section{LITTÉRATURE CITÉE}

Bernstein, J., 1902. Untersuchungen zur Thermodynamik der bioelektrischen Ströme. Pflügers Arch. ges. Pbysiol. 92, 521-562.

Cunningham, W. J., 1963. Analyse non linéaire. Dunod, Paris, 358 pp.

Gibert, R., 1952. Essai de théorie de l'excitabilité cellulaire. 1. Influx nerveux, automatismes. J. Chim. phys. 49, 448-456.

- 1953. Essai de théorie de l'excitabilité cellulaire. 2. La relation intensité-durée d'excitation. J. Chim. phys. 50, 439-444.

Helferich, F., 1963. Ion exchange. McGraw-Hill, New York, 624 pp.

Hill, A. V., 1935. Excitation and accommodation in nerve. Proc. R. Soc. (B) 119, 305-355.

HodGKIN, A. L., 1963. Conduction of the nervous impulse. Univ. pr., Liverpool, $108 \mathrm{pp}$.

Meyer, K. H. \& Sievers, J. F., 1936. La perméabilité des membranes. 1. Théorie de la perméabilité ionique. Helv. chim. Acta 19, 649-665.

Monnier, A. M., 1934. L'excitation électrique des tissus. Essai d'interprétation physique. Hermann, Paris, 326 pp.

Polissar, M. J., 1954. Physical chemistry of cell irritability and of the nervous impulse. In: The Kinetic basis of molecular biology. Ed. by F. H. Johnson, H. Eyring \& M. J. Polissar. Wiley, New York, 604-698.

RashevsKy, N., 1933. Outline of a physico-mathematical theory of excitation and inhibition. Protoplasma 20, 42-56.

- 1950. Nervous system. Mathematical theory of its functions. In: Medical physics. Ed. by O. Glasser. Year Book Publs, Inc., Chicago, vol. 2.

TEORELx, T., 1935. Diffusion effect on ionic distribution. 1. Theoretical considerations. Proc. natn. Acad. Sci. U.S.A. 21, 152-161. 


\section{Discussion suivant le discours de GIBERT}

Drischel: I believe that the two very distinctly chosen hypotheses of Dr. GrberT may be adequate to decide the question, how far the classical theory of excitation of HodgKIN, Huxley, Katz, etc. is able to explain the divergences between empirical data and theoretical prognoses.

GIBERT: Les hypothèses proposées offrent une interprétation physico-chimique des propriétés que la théorie classique postule au sujet des membranes. Il est permis d'espérer qu'elle permettra ainsi de réduire certaines des divergences signalées par le Dr. DR ISCHEL.

ROBERTS: What is your view on the hyperpolarisation following repolarisation which usually is considered to be a fatigue effect?

GIbERT: Dans le modèle proposé, l'hyperpolarisation apparaît au cours de la réaction 4 de synthèse de l'ion $\mathrm{Y}-$; elle ne traduit donc pas un phénomène de fatigue (passif) mais un phénomène de récupération (actif).

Scharf: Um voranzukommen, ist es fraglos wünschenswert, das Modell soweit wie möglich zu vereinfachen. Als Neurohistologe habe ich aber Bedenken, das Axoplasma völlig außer Ansatz zu lassen. Nach Untersuchungen von Eccles und eigenen Befunden ist sicher, daß im Axoplasma ein kompliziertes System vorliegt, in dem unter anderem durch dauernden Mitochondrienzerfall Fermente freigesetzt werden, deren Interaktion die reinen Membraneffekte erheblich modifiziert. Berücksichtigt man nur diese Möglichkeit unter vielen, dann reicht ein Modell auf reiner Membraneffekt-Basis nicht mehr aus.

GiberT: Le modèle n'est pas basé sur les seuls phénomènes de membrane, mais suppose que le "milieu intérieur», qui représente l'ensemble de la cellule, a des propriétés qui varient au cours des réactions chimiques dont il est le siège. - Les observations des histologistes signalées par le Dr. SCharf, des physiologistes et des biochimistes devraient permettre d'identifier et de localiser ces réactions.

ZERBST: Bei sogenannten axoplasmafreien Axonen ist immer noch in Membrannähe ein Axoplasma-Rest vorhanden - allerdings von geringer Schichtdicke - aber hinreichend für weitere Erregungsbildung. Die Arbeitsgruppe Schmitt (Philadelphia) konnte mit besonderen Methoden völlig axoplasamafreie Axone herstellen; diese zeigten dann aber keine Erregbarkeit.

Gibert: La réponse est la même que pour le Dr. Scharf.

LOCKER: Il est très impressionnant pour moi que le Dr. GIBERT est capable de représenter par un modèle si simple physico-chimique une multitude des phénomènes biologiques comme ils s'expriment dans la loi de sommation, la loi du tout ou rien et les caractères de l'excitation et j'en suis convaincu que la méthode proposée nous fournirait ayec certitude des résultats précieux même dans l'avenir prochain si elle avait du succès chez les physiologistes. 